3. Parmi les différentes méthodes de chauffage du lait, la pasteurisation basse n'apporte aucune modification dans la réaction; la stassanisation en diminue la sensibilité; les autres procédés de chauffage l'annihilent complètement.

4. La disparition de la précipitation spécifique s'effectue entre 70 et $75^{\circ}$, limites entre lesquelles la lactoglobuline est complètement coagulée.

L'allure de la précipitation spécifique dans le lactosérum permet donc de déceler si cette température critique a été atteinte à l'occasion de la pasteurisation du lait.

\title{
BIBLIOGRAPHIE
}

[1] J. Bondet. Annal. Inst. Pasteur, mars 1899.

[2] O. Gengou. Trav. Inst. Pasteur, 1901-1909.

[3] O. Gengou. Agglutination et précipitation spécifiques. Le traité du sang, t. II, p. 344 .

[4] J. Bordet. Traité de l'immunité dans les maladies infeetieuses, Masson, Paris, 1920.

[5] J. E. Lane-Clayfon. Milk and its hygienic relations, Longmans et Green, London, 1916.

[6] Rupp. U.S.Dep. of Agric., bull. 166, 1913.

[7] Rogers. Fundamentals of Dairy Science, Reinhold, New-York, 1935.

[8] W. L. Davies. Chemistry of Milk, Chapman et Hall, London, 1936.

[9] ВАВСоск. 12th Ann. Rep. Wisc. Agric. Exp. Sta., 1895, p. 93.

[10] Freudenrich. Revue gén. du Lait, 1905, 4, 433.

[11] Rowland. Journal Dairy Research, 1933, 5, 46.

[12] W. L. Davies. Journal Dairy Research, 1932, 4, 144.

\section{LE DOSAGE \\ DES CHLORURES PAR MICROSÉDIMENTATION. APPLICATION AU LAIT.}

\author{
par \\ RADU VLADESCO
}

Professeur de Chimie biologique. Faculté de Médecine Vétérinaire de Bucarest.

Le dosage des chlorures devient un problème délicat lorsqu'il s'agit de quantités très réduites de ces corps. En effet, en utilisant l'azotate d'argent, réactif habituel des chlorures, on arrive, avec 1 milligramme de chlorure, par la voie gravimétrique, à peser 4 milligrammes de chlorure d'argent et, par la voie volumétrique, à mesurer un volume de $1,4 \mathrm{~cm}^{3}$ de solution au cinquantième normale d'azotate d'argent. Les solutions plus étendues d'azotate d'argent ne sont pas à conseiller, parce que l'appréciation de la fin de la réaction est très difficile. A ce point de vue, il n'y a pas de différence 
entre le procédé de Mohr et celui de Charpentier-Volhard. Ces derniers temps, Geyer et Rotsch ont proposé, pour le dosage des chlorures, la méthode dont le principe est le suivant : l'azotate de mercure introduit dans une solution acide qui contient à la fois des chlorures solubles et du nitroprussiate de sodium, donne un précipité (la solution devient opalescente) seulement après la transformation totale des chlorures solubles en chlorure de mercure: Avec le chlorure de sodium, par exemple, la réaction se passe de cette façon :

$$
2 \mathrm{NaCl}+\left(\mathrm{NO}^{3}\right)^{2} \mathrm{Hg} \rightarrow 2 \mathrm{NO}^{3} \mathrm{Na}+\mathrm{Cl}^{2} \mathrm{Hg} .
$$

Cette méthode est susceptible des mêmes objections lorsqu'il s'agit de très petites quantités de chlorures.

La microsédimentation, dont nous avons exposé le principe dans deux notes insérées dans les Comptes Rendus de l'Académie des Sciences (1) permet, par contre, dè pousser plus loin la sensibilité du dosage des chlorures à l'état de chlorures d'argent. Nous employons, dans ce but, un tube à centrifuger (en verre Pyrex) de forme conique, au sommet duquel on a soudé un capillaire cylindrique ayant une lumière uniforme de quelques dixièmes de millimètre de diamètre. Le tube capillaire, après avoir été rempli avec de l'eau distillée, est fermé à son extrémité libre avec un peu de cire de Golaz (fondue à la flamme d'une veilleuse). Dans ce tube, ainsi préparé, on ramasse par centrifugation (pendant une durée et à une vitesse déterminées) les précipités résultant de quantités variées de chlorure. De cette façon, le capillaire est étalonné avec des quantités connues de chlorure d'argent (et autant que possible aussi rapprochées). Le débouchage du capillaire est effectué très facilement à l'aide d'un mince fil en tungstène.

Le nettoyage du tube, après chaque centrifugation, nécessite d'abord de l'ammoniaque (pour dissoudre le précipité de chlorure d'argent) et ensuite de l'eau distillée. Avec un tube dont le capillaire mesurait environ $0 \mathrm{~mm}$. 5 de diamètre, et à l'aide d'une solution titrée de chlorure de magnésium, nous avons obtenu les valeurs suivantes :

\begin{tabular}{|c|c|c|c|c|c|c|c|c|c|c|}
\hline $\mathrm{Cl} \ldots$ & 13,3 & 26,6 & 39,9 & 53,2 & 66,5 & 79,8 & 93,1 & 106,4 & 119,7 & 133 \\
\hline H... & 0,75 & 1,75 & 2,25 & 2,75 & 3,50 & 4,25 & 4,50 & 5,50 & 5,75 & 6,75 \\
\hline CI & 146,3 & 159,6 & 172,9 & 186,2 & 199,5 & & & & & \\
\hline H & 7,00 & 7,50 & 8,00 & 8,25 & 9,25 & & & & & \\
\hline
\end{tabular}

où $\mathrm{Cl}$ représente le chlorure (en gamma) et $\mathrm{H}$ la hauteur de la colonne

(1) Comptes Rendus Académie des Sciences, 1940, 211, 260 et 642. 
de chlorure d'argent (en milligramme). Les précautions à prendre dans l'utilisation des tubes à sédimentation sont les suivantes :

1. Les tubes doivent être bien propres afin d'empêcher l'adhérence du chlorure d'argent aux parois (l'emploi du mélange sulfochromique est tout à fait indiqué pour leur nettoyage).

2. Le capillaire ne doit pas contenir des bulles d'air dont la présence empêcherait la sédimentation régulière.

$$
*^{*} *
$$

Nous avons appliqué cette méthode au dosage des chlorures du lait de vache. Voici la technique suivie : la minéralisation de ce produit biologique, opération indispensable pour le dosage des chlorures, a été faite, ainsi que nous l'avons exposé autrefois (1), par le chauffage du lait préalablement additionné d'un excès d'azotate d'argent, dans un ballon Kjeldahl, avec de l'acide azotique concentré. Lorsque le liquide surnageant le précipité de chlorure d'argent est tout à fait clair, et après refroidissement, on ajoute un excès d'ammoniaque afin de dissoudre complètement le chlorure d'argent. Le contenu du ballon Kjeldahl est ensuite débarrassé des corps précipitables en milieu ammoniacal, par centrifugation, et le liquide séparé (ainsi que l'eau de lavage) est décanté dans le tube à sédimentation (étalonné d'avance).

On y introduit un excès d'acide azotique concentré, goutte à goutte (jusqu'à réaction nettement acide, pour reprécipiter le chlorure d'argent), et on centrifuge sous la vitesse et pendant la durée adoptées lors de l'étalonnage du tube à sédimentation. Une prise d'essai de $0 \mathrm{~cm}^{3} 1$ de lait est amplement suffisante.

Les quelques échantillons de lait, ainsi analysés, ont montré des teneurs en chlore variant entre 0 gr. 931 et $1 \mathrm{gr} .064$ par litre. Comme on le voit, ce sont des valeurs tout à fait rapprochées de celles données par $\mathrm{Ch}$. PoRcher dans ses remarquables travaux concernant la distribution des sels minéraux dans le lait.

$$
*^{*} *
$$

La fidélité de la méthode a été vérifiée pạr des analyses faites :

1. Sur des prises d'essai provenant d'un même lait.

2. Sur des échantillons de lait auxquels on avait ajouté des quantités connues de chlore. Les erreurs, quoique assez importantes, (environ $8 \%$ ), peuvent être considérées comme tolérables, étant donné que la teneur en chlore du lait varie, non seulement entre les différents individus de la même espèce, mais même chez le même individu.

(1) Comptes Rerdus de la Société de Biologie, 1925, 92, 546. 
Nous pensons que la méthode exposée pourra rendre des services utiles toutes les fois que la prise d'essai du produit biologique à analyser ne permettra pas l'application des méthodes couramment appliquées.

\title{
LE DOSAGE ÉLECTROPHOTOMÉTRIQUE DES VITAMINES $B_{1}$ ET $B_{2}$ \\ DU LAIT SANS ADSORPTION PRÉALABLE (1)
}

\author{
par
}

\author{
Mlle Andrée VINET et M. Paul MEUNIER
}

Après élimination du beurre, de la caséine, des phosphates et du lactose, on obtient, sans adsorption, un extrait de lait dans lequel on peut doser la vitamine $B_{1}$ par son azo-réaction effectuée avec et sans ferricyanure (méthode MeunierBlancpain [1]) et la vitamine $B$, par colorimétrie directe.

Les faibles concentrations sous lesquelles les vitamines $B_{1}$ et $B_{2}$ se rencontrent dans la plupart des produits naturels ont obligé les biochimistes à concentrer ces vitamines par une adsorption préalable avant de procéder à leur dosage par fluorométrie. Les difficultés techniques de cette méthode d'isolement, d'une part, et des mesures de lumière de fluorescence, d'autre part, sont bien connues.

Nous voulons montrer par suite de quelles circonstances favorables le dosage des vitamines $B_{1}$ et $B_{2}$ est possible dans le lait sans adsorption préalable et par l'emploi de l'électrophotomètre.

Après plusieurs essais, nous avons choisi le mode d'extraction suivant : la caséine et les matières grasses sont éliminées par l'acide acétique à $2 \%$. le résidu, évaporé à sec sous vide est extrait par l'alcool méthylique qui insolubilise les phosphates et le lactose et entraîne la totalité des vitamines $B_{1}$ et $B_{2}$. Dans l'extrait final, la vitamine $B_{1}$ est le seul corps diazotable et la vitamine $B_{2}$ le seul pigment mesurable avec l'écran bleu de l'appareil.

\section{Technique}

$1^{0}$ Extraction. - Placer au bain-marie bouillant à reflux $100 \mathrm{~cm}^{3}$ de lait et $100 \mathrm{~cm}^{3}$ d'acide acétique à $2 \%$. Laisser une demiheure.

Filtrer sur filtre de Buchner et rineer le filtre à l'aide de $20 \mathrm{~cm}^{3}$ d'acide acétique à $2 \%$.

Ajouter ensuite $100 \mathrm{~cm}^{3}$ d'alcool absolu et abandonner deux heures. Filtrer à nouveau. Evaporer le filtrat sous vide jusqu'à

(1) Bulletin de la Société de Chimie biologique, t. XXII, septembre-décembre 1940 , p. 556-558. Mémoire présenté à la séance du 15 octobre 1940 . 